\title{
A survey of specialist opinions on biomarker use in severe asthma in Australia: scepticism but hope?
}

\section{To the Editor:}

Recently, a survey has been published on the opinions of UK and Europeans specialists regarding the use of biomarkers in asthma [1]. The results highlight that despite the growing body of research, and the wealth of targets in blood, sputum, tissue and breath, there has been little change in the application of biomarkers to clinical practice. While the virtues of precision medicine continue to be extolled, practically there has been little enthusiasm in real-world clinics. To investigate the factors behind this and whether this holds true outside Europe, we have mirrored the approach of GaLLAGHER et al. [1] and conducted a Delphi survey of specialist physicians in Australia to gauge their opinions on the application of biomarkers in severe asthma (Figure 1).

A total of 17 responses were received from Australian specialist members of the Asthma Special Interest Group (SIG) of the Thoracic Society of Australia and New Zealand (TSANZ) from November 2017 until May 2019, with two rounds required to reach consensus. Postcode data supported the national scope of responses from New South Wales $(n=4)$, Queensland $(n=4)$, South Australia $(n=2)$, Tasmania $(n=1)$ and Victoria $(n=6)$. Respondents reported seeing a median of six patients with severe asthma per month, practicing in urban environments, and a median of 15 years of experience, with $76 \%$ practising in a public hospital. Consensus was reached on the following statements based on a majority response (agreement of $>50 \%)$ to a particular value or statement. This was achieved on all statements except that in Question 4a.

\section{Question 1}

Do you currently use biomarker based adjustment of treatment in ROUTINE clinical care (i.e. outside a clinical trial) to make adjustments of corticosteroid treatment in your clinic?

The majority of respondents (53\%) do use or would like to use biomarkers for this. The biomarkers identified as being in current use were the fraction of exhaled nitric oxide $\left(F_{\mathrm{ENO}}\right)$ and full blood eosinophil count $(\mathrm{FBC})$.

\section{Question 2}

Do you believe biomarker based adjustment of corticosteroid dose is currently the optimal way to adjust corticosteroid treatment in patients with severe asthma compared to symptoms, lung function and exacerbation history?

The majority of respondents (58\%) did not agree with this statement. Notably, a comment was made about the lack of access to biomarkers that may be hampering their use in the community.

\section{Question 3a}

For ORAL corticosteroid (OCS) treatment (i.e. daily prednisolone) what \% of patients requiring maintenance OCS do you believe would be required to be on a "lower" dose of daily OCS treatment to justify routine use of biomarker based corticosteroid treatment adjustment compared to a strategy which does not use biomarkers?

\section{@ERSpublications}

Asthma specialists are interested in adopting biomarkers into clinical practice, but more work needs to be done to support resources towards their use and provide clearer direction on this. This concern is not limited to European specialists. https://bit.ly/2WWEQXb

Cite this article as: Ramsahai JM, Simpson JL, Heaney L, et al. A survey of specialist opinions on biomarker use in severe asthma in Australia: scepticism but hope? ERJ Open Res 2020; 6: 001132020 [https://doi.org/10.1183/23120541.00113-2020].

Copyright OERS 2020. This article is open access and distributed under the terms of the Creative Commons Attribution Non-Commercial Licence 4.0. 
Question

Population

First round of survey (Delphi)

Second round of survey (Delphi)
There is a large body of research on biomarkers, and numerous options for which biomarker to use, but there has been little change in the clinical application of biomarkers to clinical practice. What are current specialist opinions on the use of biomarkers in severe asthma? Respiratory specialists involved in the care of patients with enrolment in the Thoracic Society of Australia and New Zealand and the Australia Severe Asthma Network (n=17).

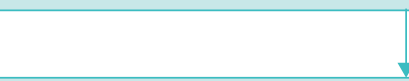

17 responses received. Results analysed for opinions on what magnitude of response would be desired out of the use of biomarkers in severe asthma. formulated from round 1 .

Final degree of consensus analysed on the questions/statements

FIGURE 1 Flow chart of survey design.

A majority of respondents thought that this number was $\geqslant 20 \%$, to justify the use of biomarker-based corticosteroid treatment adjustment.

\section{Question 3b}

What dose reduction in daily ORAL prednisolone would you regard as clinically meaningful (i.e. daily mg reduction)?

The consensus was that $\geqslant 5 \mathrm{mg}$ reduction (recognising that it may be more than this) in daily oral prednisolone would be regarded as a clinically meaningful reduction.

\section{Question 4a}

For INHALED corticosteroid (ICS) treatment (i.e. daily mcg beclomethasone dipropionate equivalent dose) what \% of patients do you believe would be required to be on a "lower" dose of ICS treatment to justify routine use of biomarker based corticosteroid treatment adjustment compared to a strategy which does not use biomarkers?

The majority of respondents (59\%) thought that this number was $\geqslant 50 \%$. Consensus was, however, not achieved on this statement. Three respondents disagreed with such a high threshold for the number of patients who would need to benefit to justify the use of biomarker-based treatment decisions. With the inclusion of these respondents, a majority of respondents did feel that biomarkers should be used in this context but at a lower threshold. One respondent did not understand the question.

\section{Question 4b}

What dose reduction in daily ICS would you regard as clinically meaningful (i.e. daily mcg beclomethasone dipropionate reduction)?

The consensus was that $\geqslant 400 \mu \mathrm{g}$ reduction in daily ICS would be regarded as clinically meaningful. 


\section{Question 5}

What \% reduction in asthma exacerbations (requiring OCS rescue therapy) do you believe would be required to justify routine use of biomarker-based corticosteroid treatment adjustment?

Respondents agreed that $\geqslant 25 \%$ reduction would be an acceptable reduction in asthma exacerbations to justify this.

\section{Question 6}

What proportion of severe asthma patients in your clinic do you believe are poorly adherent with ICS treatment as the primary clinical problem?

Respondents considered up to $40 \%$ of severe asthma patients in their clinic as poorly adherent with ICS treatment and that this was the primary clinical problem.

\section{Question 7}

What proportion of severe asthma patients in your clinic do you believe have persistent symptoms due to asthma which are NOT corticosteroid responsive?

The consensus was that $\geqslant 30 \%$ of severe asthma patients in their clinic had persistent symptoms due to asthma that were not corticosteroid responsive.

These results highlight some interesting points regarding the future of biomarker use according to Australian specialists. There has been uptake of biomarker use in the form of $F_{\mathrm{ENO}}$ and FBC. This is consistent with the availability of these tests in clinical practice and their applicability to currently available treatments for severe asthma. Promisingly, specialists seem eager to incorporate new methods to help treat this difficult to control population if there is reasonable benefit, but limits to access remain a problem.

In agreement with the European cohort, a low proportion of Australian specialists felt biomarkers were currently the optimal way to adjust corticosteroids (39\% and $42 \%$, respectively) and use them in practice (44\% and 53\%). The majority of specialists from the UK (Refractory Asthma Stratification (RASP) and non-RASP), however, do believe this (67\% and 68\%, respectively) and use them (95\% and 100\%). $F_{\mathrm{ENO}}$ and blood eosinophils would, thus, be candidate biomarkers for optimisation in clinical practice since there is little direction or consensus on how to use them in current literature. This underscores current studies looking at management algorithms that include biomarkers (MIMOSA study, ACTRN126160010 15437 [2]).

Australian respondents believed that $30 \%$ of patients had symptoms that were not corticosteroid responsive, compared to $20-25 \%$ for their UK and European counterparts [1]. This highlights a need to identify other biomarkers, such as those for non-type 2 inflammation in asthma. Aside from targeting inflammation, UK, European and Australian specialists all felt that nonadherence remains an important primary contributor to a lack of asthma control (20-50\% versus $40 \%$ of patients in Australia). This further emphasises a future role for an objective biomarker of adherence for this trait $[3,4]$.

We acknowledge the limitations of our survey; primarily that it was voluntary and sampled only a small proportion of the 239 Australian physicians that are enrolled in the Asthma SIG of TSANZ. Part of the delay in the uptake of this approach clinically relates to the limited role of biomarkers in specific subtypes of asthma, a lack of biomarkers in the noninflammatory components of asthma management and, in some centres, difficulty accessing them for clinical use. Further evidence that underlies their role in severe asthma management will be helpful to ensure their uptake in clinical practice in Australia and cement our place in the era of precision medicine.

J. Michael Ramsahai $\oplus^{1,2}$, Jodie L. Simpson ${ }^{1}$, Liam Heaney ${ }^{3}$, Nicola Gallagher ${ }^{3}$ and Peter A.B. Wark $\oplus^{1}$ ${ }^{1}$ Hunter Medical Research Institute, University of Newcastle, Newcastle, Australia. ${ }^{2}$ Dept of Medicine, Cumming School of Medicine, University of Calgary, Calgary, Canada. ${ }^{3}$ Queens University, Wellcome-Wolfson Institute for Experimental Medicine, Belfast, UK.

Correspondence: Peter A.B. Wark, HMRI, Level 2 West, Lot 1, Kookaburra Cir., New Lambton, NSW, 2305, Australia. E-mail: peter.wark@hnehealth.nsw.gov.au

Received: 7 March 2020 | Accepted: 17 March 2020

Conflict of interest: J.M. Ramsahai has nothing to disclose. J.L. Simpson has nothing to disclose. L. Heaney reports grants and other from MedImmune, grants, personal fees, non-financial support and other from Novartis, grants, 
personal fees and other from Roche/Genentech, grants, personal fees, non-financial support and other from GSK, personal fees and non-financial support from Teva, personal fees from Theravance, personal fees from Vectura, non-financial support and other from AstraZeneca, non-financial support and other from Boehringer Ingelheim, non-financial support from Chiesi, other from UK MRC Consortium for Stratified Medicine, other from Amgen, other from Janssen, outside the submitted work; and has taken part in asthma clinical trials (GlaxoSmithKline, Schering Plough, Synairgen, Novartis, and Roche/Genentech) for which his institution was remunerated. N. Gallagher has nothing to disclose. P.A.B. Wark has nothing to disclose.

\section{References}

1 Gallagher NE, Hanratty CE, Humbert M, et al. Biomarker-based corticosteroid adjustment in severe asthma: a modified Delphi consensus. ERJ Open Res 2018; 4: 00081-2018.

2 Heaney LG, Djukanovic R, Woodcock A, et al. Research in progress: Medical Research Council United Kingdom Refractory Asthma Stratification Programme (RASP-UK). Thorax 2016; 71: 187-189.

3 Heaney LG, Busby J, Bradding P, et al. Remotely monitored therapy and nitric oxide suppression identifies nonadherence in severe asthma. Am J Respir Crit Care Med 2019; 199: 454-464.

4 Ramsahai JM, King E, Niven R, et al. Serum prednisolone level as a marker of oral corticosteroid adherence in severe asthma. BMC Pulm Med 2020; in press [https://doi.org/10.21203/rs.3.rs-22760/v1]. 